\title{
Análisis de las justificaciones de los teoremas de derivabilidad en los libros de texto desde la Ley General de Educación
}

\author{
Laura Conejo, Universidad de Valladolid (España) \\ Matías Arce, Universidad de Valladolid (España) \\ Tomás Ortega, Universidad de Valladolid (España)
}

Recibido el 18 de Julio de 2015; aceptado el 13 de Octubre de 2015

\begin{abstract}
Análisis de las justificaciones de los teoremas de derivabilidad en los libros de texto desde la Ley General de Educación

Resumen

En el presente artículo se realiza una descripción exhaustiva de un marco teórico desarrollado para el análisis de las justificaciones presentadas en los libros de texto. Este marco aglutina los conceptos de esquema de prueba, prueba preformal, funciones de la demostración y otros aspectos más generales de la demostración. Con este marco se analizan las justificaciones presentes en los teoremas de Rolle y del Valor Medio en seis libros de texto, dos de cada una de las leyes de educación siguientes: Ley General de Educación, Ley de Ordenación General del Sistema Educativo y Ley Orgánica de Educación. Se presentan los resultados en forma discusiva, se comenta la aplicabilidad y la utilidad del marco teórico. Finalmente, se presentan las conclusiones y los problemas abiertos que surgen de la esta investigación.
\end{abstract}

Palabras clave. Educación; Demostración; Matemáticas; Esquema de prueba; Ley

Análise das justificações dos teoremas de derivabilidade em manuais escolares, a partir da Lei Geral da Educação

\section{Resumo}

Neste artigo faz-se uma descrição exaustiva de um marco teórico desenvolvido para a análise das justificações apresentadas nos manuais escolares. Este marco aglutina os conceitos de esquema de prova, prova pré formal, funções da demonstração e outros aspetos mais gerais da demonstração. Com este marco, analisam-se as justificações dos Teoremas de Rolle e do Valor Médio, em seis manuais escolares, presentes em duas de cada uma das Leis do Sistema Educativo seguintes: Lei Geral da Educação, Lei de Ordenação Geral do Sistema Educativo e Lei Orgânica da Educação. Apresentam-se os resultados na forma discursiva, comentando-se a aplicabilidade e utilidade do marco teórico. Finalmente, apresentam-se as conclusões e os problemas abertos levantados nesta investigação.

Palavras chave. Educação; Demonstração; Matemática; Esquema de Prova; Lei

Para citar: Conejo, L., Arce, M., \& Ortega, T. (2015). Análisis de las justificaciones de los teoremas de derivabilidad en los libros de texto desde la Ley General de Educación. Avances de Investigación en Educación Matemática, 8, 51 - 71.

(C) Sociedad Española de Investigación en Educación Matemática (SEIEM). www.seiem.es 


\section{An analysis of derivability theorems' justifications in textbooks from the 70's Spanish Education Law}

\section{Abstract}

This paper presents a description of a framework developed to carry out the analysis of the justifications of theorems that appear in textbooks. This framework brings together the concepts of proof scheme, preformal proof, functions of proofs and other more general aspects of proof. The justifications of the Rolle's Theorem and the mean value theorem in six textbooks were examined using this framework. Each of these six textbooks corresponds to one of the last three Spanish Education Laws: two to the 70's Act, two to the 90's Act and two to the Act of 2006. Results, framework's applicability and usefulness, conclusions and open problems are presented in a discursive way.

Key words. Education; Proof; Mathematics; Proof Scheme; Law

Analyse des justifications des théorèmes de dérivabilité dans les manuels depuis la Loi générale sur l'Éducation

\section{Résumé}

Cet article présente une description exhaustive d'un cadre théorique développé pour l'analyse des justifications formulées dans les manuels scolaires. Ce cadre réunit les notions de schème de preuve, preuve pre-formelle, fonctions de démonstration et d'autres aspects plus généraux de la démonstration. Il servira à analyser les justifications des théorèmes de Rolle et de la moyenne, présents dans six manuels, deux pour chacune des législations suivantes: Loi Générale sur l'Éducation, Loi organique sur l'Organisation Générale du Système Éducatif et Loi Organique relative à l'Éducation. Les résultats sont présentés sous forme de discussion où l'applicabilité et l'utilité du cadre théorique seront commentées. Enfin, seront exposées les conclusions et les questions découlant de cette recherche.

Paroles clés. Éducation; Démonstration; Mathématiques; Schème de preuve; Loi

\section{Introducción}

El libro de texto (LT) es el libro que utilizan los profesores y alumnos a lo largo de un curso escolar en el proceso de enseñanza-aprendizaje de un área de conocimiento (González, 2002). Son múltiples los autores que señalan su importancia y, además, las diversas investigaciones realizadas en el ámbito internacional sobre LT, que cada vez son más abundantes, señalan la misma idea, ya defendida por Schubring (1987): en la práctica, los LT determinan la enseñanza de un país más que los decretos de los distintos gobiernos, y tienen mayor influencia en la práctica educativa que los currículos educativos promulgados por las órdenes ministeriales. Por esta razón, para tener una visión amplia de las prácticas educativas desarrolladas en el aula, es necesario conocer qué es lo que los LT ofrecen, ya que, además, nos proporcionan información acerca del conocimiento matemático que una sociedad considera pertinente en un determinado momento histórico (González, 2002).

Teniendo en cuenta su importancia en el proceso de enseñanza-aprendizaje, nuestro interés se centra en uno de los procesos matemáticos más importantes: la demostración matemática. Desde el punto de vista de la educación matemática, Hanna (1995) considera que la demostración matemática contribuye a la comprensión de los conceptos matemáticos. La demostración contiene los métodos, herramientas, estrategias y conceptos que se necesitan para resolver problemas, y éstos elementos suponen la esencia principal de las matemáticas (Hanna \& Barbeau, 2010).

Esto nos lleva a interesarnos por su tratamiento en los LT y, por tanto, por sus repercusiones en las prácticas educativas. ¿Qué justificaciones y que explicaciones se 
ofrecen a los alumnos sobre la veracidad de los teoremas? Nuestro interés no se centra exclusivamente en la demostración formal, sino en los procesos utilizados para convencer a los alumnos, como aprendices de matemáticas, de la veracidad de las proposiciones matemáticas.

Por esta razón, desde un punto de vista didáctico, un marco teórico basado exclusivamente en la demostración formal es insuficiente para realizar un análisis exhaustivo de este proceso en los LT. Por ello, hemos desarrollado un marco teórico "ad hoc" partiendo del concepto de esquema de prueba (Harel \& Sowder, 1998; Ibañes \& Ortega, 2001) e incluyendo otros elementos que consideramos relevantes, como son las pruebas preformales (van Asch, 1993), las funciones de la demostración (de Villiers, 1993), las expresiones o el reconocimiento de procesos (Ibañes, \& Ortega, 2003). Este marco ya se ha considerado, más o menos desarrollado, en Conejo, y Ortega $(2013,2014)$ y en Conejo, Arce, y Ortega (2014, en prensa). Aquí se presenta una descripción de este marco lo suficientemente minuciosa como para poder ser aplicado a otros conceptos o niveles, y se aplica al análisis de las justificaciones de dos teoremas de derivabilidad: el Teorema de Rolle (TR) y el Teorema del Valor Medio (TVM), que se enuncian en los LT de COU de la Ley General de Educación (LGE) y de $2^{\circ}$ de Bachillerato de la Ley de Ordenación General del Sistema Educativo (LOGSE) y de la Ley Orgánica de Educación (LOE). Los objetivos de nuestro trabajo son los siguientes:

- Adaptar un marco teórico ad hoc para analizar el tratamiento de los LT en los TR y TVM.

- Analizar el tratamiento de los LT de dos editoriales en tres periodos legislativos diferentes (LGE, LOGSE y LOE) atendiendo a los siguientes aspectos: esquemas de prueba utilizados, reconocimiento de procesos, orden de presentación, expresiones utilizadas, rigor y formalismo matemático, uso de gráficas y funciones que reflejan las justificaciones utilizadas.

\section{Descripción del marco}

En primer lugar, partimos del concepto de esquema de prueba que Harel y Sowder (1998) definen desde la perspectiva del alumno (esquema de prueba personal) pero cuyas características son útiles para nuestra investigación. Como ya hemos mencionado anteriormente, nuestro objetivo no consiste en analizar únicamente la demostración matemática desde un punto de vista matemático, sino que consideramos una visión didáctica, más amplia, del proceso, y tenemos en cuenta cualquier procedimiento que para el alumno justifique el teorema y las funciones asociadas.

El concepto de esquema de prueba se ha utilizado con éxito en otros trabajos de investigación (Conejo \& Ortega, 2013; Conejo et al., 2014; Conejo \& Ortega, 2014). En concreto, en el trabajo de Conejo y Ortega (2014) se hace una descripción de un marco teórico basado en los esquemas de prueba presentes en los LT, en las pruebas preformales, y de las otras categorías que completan este marco (técnicas utilizadas, funciones de la demostración, reconocimiento de procesos, expresiones que utiliza y consideraciones globales). En ese trabajo también se presentaron los resultados obtenidos de la aplicación del marco a los teoremas de continuidad de los LT de COU (LGE) y $2^{\circ}$ de Bachillerato (LOGSE y LOE) de cuatro editoriales diferentes. Algunas de las hipótesis de las que partimos (desaparición de la demostración matemática en los LT de las nuevas legislaciones) ya fueron comprobadas en aquella ocasión en los tópicos señalados, pero limitarse a un concepto puede llevarnos a interpretaciones 
sesgadas, pues pudiera suceder que la demostración se usara de forma diferente en otros tópicos. Por otro lado, la presentación de este marco teórico en algunos congresos (Conejo et al., 2014, en prensa) y su aceptación por la comunidad investigadora garantiza la idoneidad del mismo para la investigación que aquí se describe, máxime si se tiene en cuenta que se ha reformulado para que se adapte mejor a la finalidad de nuestro trabajo.

Además, la utilización del concepto de esquema de prueba ya ha sido utilizado por otros autores, aunque bajo otras denominaciones como la de modos de razonamiento (Stacey \& Vincent, 2009). Estos autores realizan un análisis de LT australianos de octavo curso (13-14 años) clasificando las justificaciones en distintos modos de razonamientos. Los modos de razonamiento que definen son los siguientes: llamada a la autoridad, analogía cualitativa, concordancia de una regla con un modelo, demostración experimental, deducción usando un modelo, deducción usando un caso específico y deducción usando un caso general. También Dolev y Even (2015) utilizan estas categorías en LT israelitas de séptimo curso (12-13 años), que son similares a las que nosotros hemos adoptado pero que no se adaptan tan bien a nuestra investigación debido a que los estudios antes descritos son de niveles educativos inferiores al nuestro, y el rigor matemático posible a los 12 o 14 años es diferente que a los 17 o 18 .

La definición de esquema de prueba personal dada por Harel y Sowder (1998) es la siguiente: lo que constituye persuasión y convencimiento para esa persona, entendiendo como convencimiento el proceso utilizado por un individuo para eliminar sus dudas sobre la veracidad de una afirmación y como persuasión el proceso utilizado por un individuo para eliminar las dudas de otros sobre la veracidad de una afirmación (p. 244, traducido al castellano).

En esta definición juega un papel importante la subjetividad de la persona, y los esquemas de prueba de dicha persona dependen de ella misma y de los procedimientos que considera como válidos para justificar un determinado teorema, y no de las características del procedimiento matemático en sí. Sin embargo, las categorías de clasificación de los esquemas de prueba personales se han definido atendiendo a las características de dichos procedimientos. Son las diferencias entre dichas características las que permiten clasificar los procedimientos justificativos presentes en los LT. Definimos esquema de prueba (EP) de un libro de texto (LT) como lo que el libro de texto muestra que podría constituir persuasión y convencimiento para un posible lector, en este caso, un estudiante de matemáticas del nivel para el que está diseñado el libro, entendiendo como convencimiento el proceso impreso en el LT que podría eliminar las dudas del posible lector sobre la veracidad de una afirmación y como persuasión, el proceso del libro, con el cual, el posible lector podría eliminar las dudas de otros sobre la veracidad de una afirmación.

Teniendo en cuenta esta adaptación, y el objetivo de nuestro análisis, hemos descrito nuestras propias categorías. Éstas provienen de aquellas establecidas por Harel y Sowder (1998) y desarrolladas por Ibañes y Ortega (2001). Sólo hemos considerado aquellas que creemos que tienen relevancia en nuestro trabajo. Por ejemplo, no consideramos los EP autoritarios (el alumno se convence de la veracidad por la autoridad del profesor) porque esta categoría no se refleja en los LT. Por otro lado, hemos añadido otra categoría que nos ha parecido de interés en el análisis que nos ocupa, y que proviene del concepto de prueba preformal de van Asch (1993). Teniendo en cuenta estas reflexiones, se consideran las categorías de EP de los LT que se encuentran en Conejo y Ortega (2014): 
1. No se hace ningún tipo de justificación del enunciado (EP0).

2. EP inductivo de un caso (EPi1): se trata de convencer de la validez de una conjetura mediante la evaluación cuantitativa de un caso particular.

3. EP inductivo de varios casos (EPiV): como en el caso anterior, pero se realiza con varios casos particulares.

4. EP inductivo sistemático (EPiS): como en los casos anteriores, pero los ejemplos particulares se eligen de forma organizada, cubriendo las posibles diferentes casuísticas.

5. EP transformacionales $(\mathrm{EPt})$ : se realizan transformaciones de imágenes o signos por medio de la deducción.

6. EP axiomáticos $(\mathrm{EPa})$ : se realiza la demostración a partir de axiomas, entendiendo como tales los enunciados primarios y los resultados que se han deducido previamente mediante las correspondientes demostraciones.

7. Pruebas preformales (PP): se incluye como EP por su carácter de convencimiento y persuasión. Refleja la esencia de una demostración formal (EPt y EPa) y, por tanto, hereda el carácter axiomático y transformacional de estos EP.

A continuación se muestran algunos ejemplos que permitan una mejor comprensión del uso de estas categorías. Los EP inductivos de uno o varios casos o sistemáticos tienen la misma esencia y sólo difieren en el número de casos utilizados para justificar el teorema (uno o varios) y la forma de elegirlos (intento de cubrir todas las opciones posibles). Para ser considerado dentro de esta categoría, no es suficiente con mostrar ejemplos que cumplan el teorema o ejemplos en los que se muestre su aplicación; es necesario que en el libro de texto aparezca alguna indicación, o esté presentado de tal forma que permita inducir el enunciado a partir de los ejemplos que se muestran. La figura 1 muestra un ejemplo de EP inductivo de 1 caso; se razona sobre un ejemplo gráfico concreto en el que, según los autores del LT, debe haber un punto con tangente horizontal. Se limitan a marcar los puntos donde la tangente es horizontal (máximos y mínimos relativos), aunque no la dibujan, y no se indica qué resultado se aplica para asegurar la existencia de dichos puntos (en la gráfica es evidente). Sin embargo, mediante este caso concreto, los autores pretenden convencer a los lectores de que el teorema es cierto (llaman justificación al razonamiento e indican que es algo evidente).

Justificación: El teorema dice algo evidente desde el punto de vista gráfico. Si se cumplen las hipótesis del teorema debe haber un punto de la gráfica correspondiente a I con tangente horizontal.

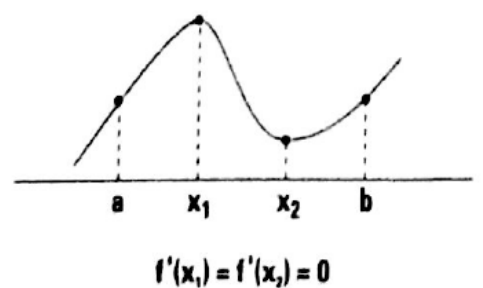

Figura 1. Ejemplo de esquema de prueba inductivo de 1 caso (EPi1) en un LT. $2^{\circ}$ de Bachillerato, LOGSE (Vicens-Vives, 1999, p. 261) 
Los EP axiomáticos son las demostraciones formales y los EP transformacionales suelen aparecer en ellos. En ocasiones, se encuentra que un procedimiento puede clasificarse en ambas categorías, pero para facilitar su recuento y tratamiento inicial, la clasificaremos en aquella que sea predominante. Un ejemplo de esta situación es la justificación del teorema de Cauchy (generalización del teorema del valor medio). En la Figura 2 los autores establecen una función auxiliar (parte transformacional de la demostración) a la que aplican el TR (parte axiomática de la demostración).

Para hacer la demostración se considera la función:

$$
h(x)=g(x)[f(b)-f(a)]-f(x)[g(b)-g(a)]
$$

Como $f$ y $g$ son funciones continuas en $[a, b]$ y derivables en $(a, b)$, también lo es $h$. Además:

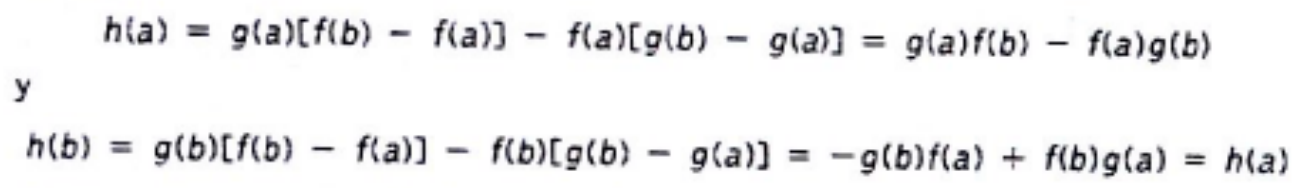

$$
\frac{f(b)-f(a)}{g(b)-g(a)}=\frac{f^{\prime}(c)}{g^{\prime}(c)}
$$

Escrito de esta manera es fácil observar que el teorema del valor medio es una consecuencia del teorema del valor medio generalizado que se obtiene tomando $g(x)=x$.

Figura 2. Ejemplo de EP transformacional (EPt) y axiomático (EPa) del teorema de Cauchy. $2^{\circ}$ de Bachillerato, LOGSE (SM, 2001, p. 298).

Para demostrar el TVM, se puede realizar un giro de los ejes de coordenadas de forma que los valores de la función en los extremos del intervalo coincidan para aplicar posteriormente el TR (Figura 3). En ambos casos se combinan ambas categorías (EP axiomático y transformacional), pero vamos a clasificarlas como EPt porque la clave de la demostración es el paso transformacional.

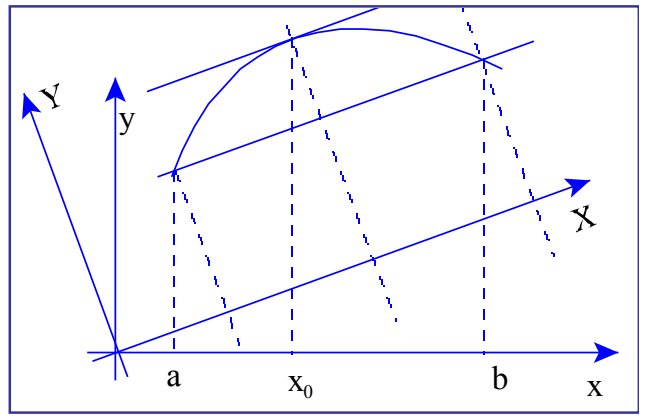

Figura 3. Transformación geométrica que justifica la aplicación del TR para demostrar el TVM. 
En este teorema se podría utilizar una prueba axiomática y transformacional, pero con predominio del EP axiomático como la que muestra la Figura 4 (y que computaríamos como $\mathrm{EPa}$, aunque matizásemos que tiene ambos tipos). Aquí, la función no se transforma en otra, sino que se razona con un elemento que tiene identidad propia: la recta secante que pasa por los puntos $(a, f(a))$ y $(b, f(b))$.

\section{Demostración}

Consideremos los puntos $A(a, f(a))$ y $B(b, f(b))$. La cuerda que une $A$ y $B$ tiene pendiente:

$$
m=\frac{f(b)-f(a)}{b-a}
$$

Consideremos la función $\gamma(x)$ obtenida restando a $f(x)$ la ordenada en el punto $x$ de la cuerda que une $A$ y $B$ :

$$
\gamma(x)=f(x)-\frac{f(b)-f(a)}{b-a} \cdot x
$$

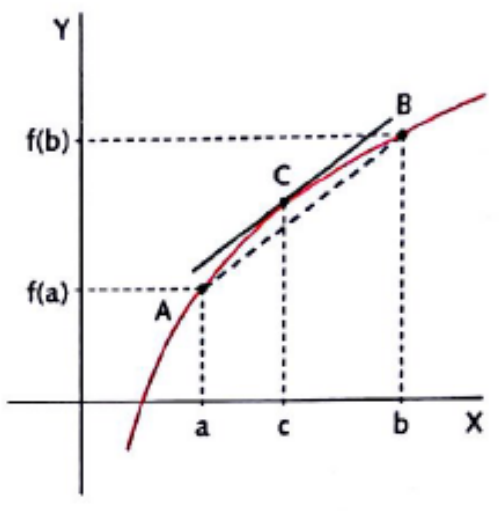

Puedes comprobar que se cumple $\gamma(a)=\gamma(b)$. Por tanto, la función $\gamma(x)$ verifica todas las hipótesis del teorema de Rolle, luego existe al menos un punto $c \in(a, b)$, tal que:

$$
\gamma^{\prime}(c)=0 \Rightarrow \gamma^{\prime}(c)=f^{\prime}(c)-\frac{f(b)-f(a)}{b-a}=0 \Rightarrow f^{\prime}(c)=\frac{f(b)-f(a)}{b-a}
$$

\section{como queríamos demostrar.}

Figura 4. EP axiomático y transformacional del TVM. $2^{\circ}$ de Bachillerato, LOE (VicensVives, 2009, p. 232).

Por último, una prueba preforma de este teorema consistiría en realizar las justificaciones anteriores, pero utilizando una función concreta en lugar de la genérica $\mathrm{f}(\mathrm{x})$. Se muestra a continuación un ejemplo:

Sea $f(x)=x^{2}$ y el intervalo $[1,2]$. La recta que pasa por $\mathrm{A}=\left(1,1^{2}\right)$ y $\mathrm{B}=\left(2,2^{2}\right)$ tiene pendiente:

$$
m=\frac{2^{2}-1^{2}}{2-1}=\frac{4-1}{2-1}
$$

Consideremos la función $g(x)$ obtenida mediante la diferencia entre $f(x)$ y la ordenada en el punto $x$ de la recta que une A y B:

$$
g(x)=f(x)-1-\frac{4-1}{2-1} \cdot(x-1)=x^{2}-1-\frac{4-1}{2-1} \cdot(x-1)=x^{2}-3 x+2
$$

Se puede comprobar que $g(1)=g(2)=0 \mathrm{y}$, por tanto, la función $g(x)$ cumple las hipótesis del $\mathrm{TR}$, luego existe un punto $c \in(1,2)$ que verifica:

$$
g^{\prime}(c)=0 \Rightarrow g^{\prime}(c)=f^{\prime}(c)-\frac{4-1}{2-1}=2 \cdot c-3=0
$$

Así, resulta, $c=1,5$. 
Como se observa, todos los razonamientos de la demostración formal aparecen en el proceso con la función $f(x)=x^{2}$, pero ésta permite realizar un gráfico preciso y localizar el punto en el que se verifica el teorema, lo que facilita la comprensión del mismo y cómo se aplica. Este tipo de justificaciones, poco usadas, pueden ser muy útiles en estos niveles educativos debido a que permiten a los estudiantes entender un razonamiento deductivo sin realizar la abstracción que supone utilizar un caso general.

Por otra parte, siguiendo el ejemplo de Ibañes y Ortega (1997), se van a clasificar las justificaciones atendiendo a las técnicas empleadas en la demostración, esto es, en función del tipo de enunciado, del método utilizado, del estilo y del modo.

- Tipo: la estructura lógica del enunciado influye en el tipo de EP utilizado. Se pueden clasificar atendiendo a: la implicación (condición necesaria, condición suficiente, y necesaria y suficiente), al cuantificador (universal -de existencia simple, imposibilidad, y existencia y unicidad-). Aunque las categorías anteriores podrían cubrir todas las formas de enunciar un teorema, el lenguaje permite reformular los enunciados de forma que no se ajuste completamente a ninguna de ellas. Por esta razón, Ibañes y Ortega (1997) añaden dos tipos más: de hipótesis y tesis mezcladas y de hipótesis implícita.

- Método: según los procedimientos lógicos utilizados en el EP: por silogismo, por casos, por reducción al absurdo, por inducción completa, constructivo, ya sea de ejemplo o contraejemplo, por analogía y por dualidad.

- Estilo: según los procedimientos matemáticos propios de las áreas de las matemáticas. En este caso, el estilo más habitual será el propio del Análisis Matemático y puede haber dos tipos: global, cuando se aplican teoremas y local, si realiza una discusión en un entorno. También puede haber estilos gráficos, utilizando coordenadas, y algebraicos.

- Modo: en función del procedimiento de exposición. Puede ser sintético o directo (se argumenta a partir de conceptos básicos), y analítico o indirecto (se utilizan otros elementos ya establecidos).

También se consideran qué funciones de la demostración de entre las enunciadas por de Villiers (1993) se reflejan en las justificaciones aquí analizadas: funciones de verificación, explicación, sistematización, comunicación y descubrimiento. De ellas, será especialmente interesante la función de explicación, por ser la más valorada por los alumnos (Ibañes \& Ortega, 2003).

Otros aspectos incluidos en este marco teórico, sobre los que hicimos referencia en Conejo y Ortega (2014), son: el reconocimiento de procesos (indicadores que se encuentren en los LT que puedan facilitar o dificultar dicho reconocimiento), las expresiones utilizadas (aspectos asociados al lenguaje y a los sistemas de representación -Janvier, 1987-), y algunas consideraciones globales (si explica el proceso, si explica la significación, los pasos clave,...).

Para enfatizar en el reconocimiento de procesos, se observará si se diferencia explícitamente el enunciado de la demostración (etiquetas, indicaciones, cambio en la tipografía,...). Por último, se revisará si, en los casos en los que se justifiquen dichos resultados, se señalan otras posibles pruebas. 


\section{Metodología. Aplicación del marco a los teoremas de derivabilidad}

Tal y como se describe en Conejo y Ortega (2014), nuestro trabajo tiene cierto carácter histórico, razón por la que se ha utilizado el método histórico de investigación en educación (Ruiz-Berrio, 1976). Este método consta de cuatro fases (heurística, crítica, hermenéutica y exposición), que aparecen descritas en Conejo y Ortega (2014) y en Conejo et al. (2014). No obstante, para precisar las acciones de cada etapa y adaptarlo a nuestro trabajo, se ha combinado con las etapas de la metodología de investigación en educación de Fox (1981).

Se ha considerado una muestra de tres libros de dos editoriales, uno de cada periodo legislativo considerado. Tal y como se justifica en Conejo y Ortega (2014), los documentos curriculares correspondientes a las tres legislaciones indicadas dejan claro que la demostración matemática y las justificaciones deben formar parte de las matemáticas de estos niveles.

Los seis libros analizados son dos ejemplares de COU, dos de $2^{\circ}$ de Bachillerato LOGSE y dos de $2^{\circ}$ de Bachillerato LOE, de las editoriales Vicens-Vives y SM. Para facilitar la redacción y lectura del texto, vamos a referirnos a cada ejemplar analizado con el código: Editorial Año. La Tabla 1 muestra el resumen de la muestra analizada.

Tabla 1. Muestra de los LT analizados en el presente trabajo.

\begin{tabular}{cccc}
\hline & LGE & LOGSE & LOE \\
\hline SM & SM 1980 & SM 2001 & SM 2010 \\
Vicens-Vives & VV 1979 & VV 1999 & VV 2009 \\
\hline
\end{tabular}

En concreto, se analizarán el TR y el TVM en los seis textos seleccionados. Dada la diversidad de nomenclaturas encontrada, a continuación presentamos unos enunciados de referencia de los teoremas analizados, siguiendo a Spivak (1981):

TR: si $f$ es continua sobre $[a, b]$ y derivable sobre $(a, b)$, y $f(a)=f(b)$, entonces existe un número $x$ en $(a, b)$ tal que $f^{\prime}(x)=0$. (Spivak, 1981, p. 247)

TVM: si $f$ es continua en $[a, b]$ y derivable en $(a, b)$, entonces existe un número $x$ en $(a, b)$ tal que $f^{\prime}(x)=[f(b)-f(a)] /(b-a)$. (Spivak, 1981, p. 248)

En este texto, el orden de los teoremas es el que figura aquí y la justificación del TVM se basa en el TR.

\subsection{Aplicación de los esquemas de prueba}

La Tabla 2 muestra los esquemas de prueba utilizados en las justificaciones de los teoremas seleccionados. En ella se observa que el EP predominante para el TR es el EP axiomático, y para el TVM se utiliza tanto un EP axiomático puro como EP axiomático y transformacional. No obstante, las pruebas utilizadas no son siempre las mismas, e incluso en aquellas similares, hay algunos rasgos diferenciadores. Además, Vicens-Vives (1999) utiliza EP inductivos de 1 caso y SM (2001), considera un EP inductivo de varios casos previo al enunciado del teorema y su justificación axiomático-transformacional. 
Tabla 2. Clasificación de los esquemas de prueba de TR y TVM en los LT de la muestra analizada.

\begin{tabular}{|c|c|c|c|}
\hline & & T. Rolle & T. Valor medio \\
\hline \multirow[t]{2}{*}{ LGE } & SM 1980 LGE & EP axiomático & EP axiomático \\
\hline & VV 1979 LGE & EP axiomático & EP axiomático \\
\hline \multirow[t]{2}{*}{ LOGSE } & SM 2001 LOGSE & EP axiomático & $\begin{array}{l}\text { EP inductivo de varios casos; EP } \\
\text { axiomático y transformacional }\end{array}$ \\
\hline & VV 1999 LOGSE & EP inductivo de 1 caso & EP inductivo de 1 caso \\
\hline \multirow[t]{2}{*}{ LOE } & SM 2010 LOE & EP axiomático & $\begin{array}{l}\text { EP axiomático y } \\
\text { transformacional }\end{array}$ \\
\hline & VV 2009 LOE & EP axiomático & $\begin{array}{l}\text { EP axiomático y } \\
\text { transformacional }\end{array}$ \\
\hline
\end{tabular}

En el caso del TR, SM (1980, 2001, 2010) y Vicens-Vives (2009) presentan una justificación basada en los siguientes pasos: por el teorema de Weierstrass, se asegura que en el intervalo $[a, b]$ se alcanzan el máximo y el mínimo absolutos. A continuación, se realiza una discusión por casos. Si ambos extremos se alcanzan en los extremos del intervalo, la función es constante y en todos los puntos del intervalo la derivada se anula. Si alguno de los extremos se encuentra en el interior del intervalo, o bien se justifica que la función derivada se anula en dicho punto (SM, 1980) o bien se recurre a un resultado anterior: "la derivada de una función se anula en los extremos relativos" (el resto). Vicens-Vives (1979) presenta el TR después del TVM, indicando en el propio enunciado que se cumple por aplicar la fórmula del TVM. Por su parte, Vicens-Vives (1999), que muestra un EP inductivo de un caso, presenta una gráfica de una función que cumple las hipótesis del teorema (Figura 1), indica que hay puntos en los que la tangente a la gráfica es horizontal, y señala dichos puntos.

En el caso del TVM, SM (1980) y Vicens-Vives (1979) utilizan un EP axiomático. En el primer caso, el TR se presenta después del teorema de Cauchy (como teorema generalizado del valor medio), y se utiliza este último para justificar el primero, considerando el caso particular en que una de las funciones es la identidad. VicensVives (1979) se apoya en el desarrollo de Taylor de la función para una derivada continua. En ese caso, el desarrollo de Taylor de la función da lugar directamente al enunciado del teorema. SM (2001, 2010) y Vicens-Vives (2009) utilizan un EP axiomático y transformacional: consideran un elemento auxiliar, la recta secante que pasa por los puntos $(a, f(a))$ y $(b, f(b))$ (siendo $a$ y $b$ los extremos del intervalo), y se construye la función diferencia entre la función $f$ (del enunciado) y dicha recta secante (parte transformacional de la justificación). Por último, se aplica el TR (parte axiomática de la prueba). Además, en SM (2001) el enunciado del teorema va precedido de un EP inductivo de varios casos, en el que se muestran varias gráficas, las rectas secantes que pasan por los extremos del intervalo, y rectas tangentes a las gráficas con la misma pendiente que las rectas secantes (Figura 5). Estas gráficas son explicativas porque justifican la elección de la recta secante para realizar la demostración. En Vicens-Vives (1999) se muestra un EP inductivo de un caso y, de forma similar, considera la secante determinada por $(a, f(a))$ y $(b, f(b))$. También utiliza la imagen para justificar que debe existir una tangente paralela a la secante descrita. 


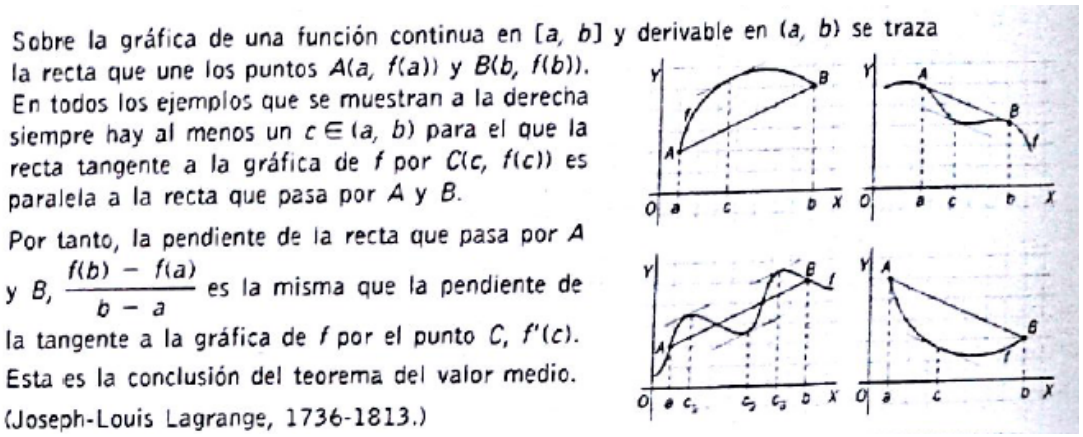

Figura 5. EP inductivo de varios casos (SM, 2001, p. 297).

No obstante, aunque se hayan utilizado justificaciones similares en algunos textos, existen diferencias en la completitud de dichas justificaciones, en el lenguaje o los sistemas de representación utilizados, en el acompañamiento o no de gráficas y en su selección. Todo ello hace que los procesos de justificación tengan matices diferentes. El resto de categorías recogen estos detalles y nos permiten diferenciar entre dos justificaciones aparentemente similares, y seleccionar aquellos aspectos que consideramos más valiosos, desde el punto de vista de la enseñanza, para plantear nuestra propia propuesta.

\subsection{Aplicación de las técnicas empleadas}

Aquí se analizan las técnicas empleadas en cada LT y teorema: tipo de enunciado, método empleado, estilo y modo. Empezando por el enunciado, todos los LT denotan el TR por dicho nombre, pero no ocurre lo mismo con el TVM: utilizan este nombre SM (2001) y Vicens-Vives (1999), ambos de LOGSE; en SM (2010) y en VicensVives (2009), ambos de LOE, aparece como "TVM o de Lagrange" (aunque en el SM lo indica en el texto); los LT de la LGE le designan como teorema de los incrementos finitos en SM (1980) y teorema del incremento finito en Vicens-Vives (1979). En las Tablas 3 y 4 se recogen los enunciados de los teoremas tal como son formulados en los LT de nuestra muestra.

Tabla 3. Enunciados del TR en los LT de la muestra.

\begin{tabular}{|c|c|c|}
\hline & & T. Rolle \\
\hline LGE & SM 1980 LGE & $\begin{array}{l}\text { Si una función es continua en el intervalo cerrado }[a, b] \text { y derivable } \\
\text { en el intervalo abierto }(a, b) \text { y además } f(a)=f(b) \text { entonces existe, al } \\
\text { menos, un punto } \alpha \in(a, b) \text { donde la derivada de la función se anula, } \\
\text { es decir, } f^{\prime}(\alpha)=0 . \\
\text { Si } h \neq 0 \text { y } f\left(x_{0}+h\right)=f\left(x_{0}\right) \text {, entonces existe un punto del intervalo }\left[x_{0} \text {, }\right. \\
\left.x_{0}+h\right] \text { en el que la derivada de } f \text { se anula, ya que la fórmula del valor } \\
\text { medio indica que } f^{\prime}(c)=0 \text { para } c \in\left[\mathrm{x}_{0}, x_{0}+h\right] .\end{array}$ \\
\hline LOGSE & $\begin{array}{l}\text { SM } 2001 \text { LOGSE } \\
\text { VV } 1999 \text { LOGSE }\end{array}$ & $\begin{array}{c}\text { Sea } f \text { una función continua en }[a, b] \text { y derivable en }(a, b) \text {. Si } f(a)= \\
f(b) \text { existe algún punto } c \in(a, b) \text { tal que } f^{\prime}(c)=0 \text {. } \\
\text { Sea } \mathrm{I}=[a, b] \text { un intervalo contenido en Dom } f \text {. Si f es continua en } \\
{[a, b] \text {, derivable en }(a, b) \text { y } f(a)=f(b) \text { entonces existe un número } \alpha} \\
\text { en el interior de I, tal que } f^{\prime}(\alpha)=0 \text {. }\end{array}$ \\
\hline LOE & SM 2010 LOE & $\begin{array}{l}\text { Si } f \text { es continua en un intervalo cerrado }[a, b] \text { y derivable en el } \\
\text { intervalo abierto }(a, b) \text {, y además } f(a)=f(b) \text {, entonces existe al } \\
\text { menos un punto } c \text { del intervalo }(a, b) \text { con } f^{\prime}(c)=0 \text {. } \\
\text { Sea } f \text { una función continua en }[a, b] \text { y derivable en }(a, b) \text {. Si } f(a)= \\
f(b) \text {, entonces existe al menos un punto } c \in(a, b) \text { tal que } f^{\prime}(c)=0 \text {. }\end{array}$ \\
\hline
\end{tabular}


En general, todos los LT utilizan enunciados con formato similar al de Spivak (1981) para los dos teoremas, exceptuando el LT de Vicens-Vives (1979). Los enunciados del TR son de condición suficiente, y la hipótesis y la tesis están diferenciadas y enunciadas de forma explícita. Algunos enunciados no permiten deducir que pueda existir más de un punto que cumpla las condiciones del teorema. Los enunciados que sí lo hacen utilizan formulaciones del tipo "existe, al menos, un punto" o "existe algún". Utilizan estas expresiones SM (1980, 2001, 2010) y VicensVives (2009) en el TR y en el TVM. El enunciado de Spivak no contempla esta pluralidad.

Tabla 4. Enunciados del TVM en los LT de la muestra.

\begin{tabular}{|c|c|c|}
\hline & & Teorema del Valor medio \\
\hline LGE & SM 1980 LGE & $\begin{array}{l}\text { Teorema de los incrementos finitos: si } f(x) \text { es una función continua } \\
\text { en el intervalo cerrado }[a, b] \text { y derivable en el abierto }(a, b) \text {, } \\
\text { entonces existe, al menos, un punto } \alpha \in(a, b) \text { tal que } f^{\prime}(\alpha)=[f(b)- \\
f(a)] /(b-a) \text {. } \\
\text { Teorema del incremento finito: la pendiente de la secante que une } \\
\text { los puntos }\left(x_{0}, f\left(x_{0}\right)\right) \text { y }\left(x_{0}+h, f\left(x_{0}+h\right)\right) \text { es igual a la de la tangente en } \\
\text { un punto intermedio }(c, f(c)) \text {. }\end{array}$ \\
\hline LOGSE & SM 2001 LOGSE & $\begin{array}{l}\text { TVM: si f es una función continua en }[a, b] \text { y derivable en }(a, b) \text {, } \\
\text { existe algún } c \text { del intervalo }(a, b) \text { para el que se cumple: }[f(b)- \\
\qquad f(a)] /(b-a)=f^{\prime}(c) \text {. } \\
\text { TVM: sea } \mathrm{I}=[a, b] \text { un intervalo contenido en Dom } f \text {. Si } f \text { es } \\
\text { continua en }[a, b] \text {, derivable en }(a, b) \text { entonces existe un } \alpha \text { en el } \\
\text { interior de I tal que } f^{\prime}(\alpha)=[f(b)-f(a)] /(b-a) \text {. }\end{array}$ \\
\hline LOE & SM 2010 LOE & $\begin{array}{l}\text { TVM: si } f \text { es continua en el intervalo cerrado }[a, b] \text { y derivable en } \\
(a, b) \text {, entonces existe un número } c \in(a, b) \text { con } f^{\prime}(c)=[f(b)-f(a)] /(b \\
-a) \text {. } \\
\text { TVM o de Lagrange: Si la función } f \text { es continua en }[a, b] \text { y } \\
\text { derivable en }(a, b) \text {, entonces existe al menos un punto } c \epsilon(a, b) \text {, tal } \\
\text { que } f^{\prime}(c)=[f(b)-f(a)] /(b-a) \text {. }\end{array}$ \\
\hline
\end{tabular}

Los enunciados presentados por Vicens-Vives (1979) son diferentes. En el enunciado del TVM (teorema del incremento finito en el LT) se utilizan conceptos geométricos y en el enunciado del TR, que es similar al habitual, los extremos del intervalo están expresados en función de un punto y un parámetro, en lugar de dos valores independientes. Además, el orden considerado es el contrario al habitual.

Atendiendo al método (procedimientos lógicos utilizados en la justificación), encontramos los siguientes: para el TR, los LT que han presentado la justificación axiomática basada en la aplicación del teorema de Weierstrass y la existencia de extremos, lo hacen utilizando el silogismo de manera general y una discusión por casos. En SM (2010) y Vicens-Vives (2009) se distinguen dos casos: que tanto el mínimo como el máximo se alcancen en los extremos del intervalo o que alguno de ellos se alcance en el interior. En SM $(1979,2001)$ se distinguen tres casos: que el máximo y el mínimo se alcancen en los extremos del intervalo, que el máximo se alcance en un punto que no es un extremo del intervalo o que el mínimo se alcance en un punto que no es un extremo del intervalo. Aunque las dos últimas condiciones son equivalentes a la condición de que se alcance algún extremo en el interior del intervalo, es más explicativa la segunda opción, pues muestra todas las posibilidades sin dejar ningún ejercicio para el lector. Finalmente, Vicens-Vives (1979) realiza un 
silogismo en el mismo enunciado a partir del teorema anterior para establecer el resultado. No se clasifican las pruebas de Vicens-Vives (1999) pues al ser EP inductivos de 1 caso, no hay procedimiento lógico en la justificación.

Para el TVM, en todos los tipos de pruebas se ha utilizado el silogismo, aunque en los EP axiomáticos y transformacionales aparece una construcción: la función auxiliar que permite establecer el resultado.

Todos los EP inductivos de la muestra atienden a un estilo gráfico, ya que se apoyan en las gráficas para razonar sobre la validez del teorema. En el TR, casi todos los LT que utilizan EP axiomático atienden a un estilo del Análisis Matemático (AM) global, al limitarse a la aplicación de teoremas. Tan sólo SM (1980) combina el estilo de AM global con una discusión propia del AM local, ya que estudia el comportamiento de la derivada en un entorno de un extremo relativo de la función. Para el TVM, los EP axiomáticos y transformacionales combinan el estilo de las coordenadas (formulación de la recta secante determinada por $(a, f(a))$ y $(b, f(b))$, y construcción de la nueva función auxiliar) y del AM global, ya que aplica el TR a la función auxiliar construida. Por su parte, el EP axiomático utilizado en SM (1980) atiende a un estilo del AM global, ya que aplica el teorema de Cauchy, y el que muestra Vicens-Vives (1979) tiene un estilo más algebraico, ya que argumenta a partir de igualdades algebraicas.

Por último, el procedimiento de exposición (modo) utilizado en todos los razonamientos es analítico, ya que se utilizan resultados establecidos previamente y no sólo los conceptos básicos.

\subsection{Aplicación de la categoría de reconocimiento de procesos}

Todos los LT etiquetan los teoremas seleccionados como "teoremas", es decir, por parte del LT se reconoce que el enunciado propuesto es un resultado matemático, no evidente y que requiere de justificación. No obstante, para un alumno la etiqueta de teorema no tiene por qué significar una necesidad de justificación si no se le ha indicado previamente. En nuestra muestra, únicamente Vicens-Vives (2009) etiqueta las demostraciones con la referencia explícita "demostración", aunque no explica que el proceso expuesto a continuación es una demostración del teorema enunciado. En SM $(2001,2010)$, aunque no se etiqueta el proceso, se hacen indicaciones en el texto del tipo "para demostrar el teorema" o "para hacer la demostración", lo que indica que se reconoce dicho procedimiento como una justificación. En Vicens-Vives (1999) se utilizan sendos EP inductivos de 1 caso para estos teoremas, el razonamiento está precedido de la palabra "justificación" lo que parece indicar que la intención reflejada en el LT es justificar el teorema enunciado, aunque no se considere como una demostración. Por su parte, en ninguno de los LT de la LGE se indica que el razonamiento descrito sea una demostración. Además, hay que señalar que en ninguno de los LT se señala el final de la demostración o justificación utilizada.

En cuanto a las consecuencias, ninguno de los LT hace referencias a las consecuencias de haber justificado el resultado (que no son necesarias comprobaciones posteriores o que no se pueden localizar contraejemplos). Sin embargo, SM (2001) y Vicens-Vives (1999) indican la necesidad de las hipótesis del teorema para que sea cierto, mostrando ejemplos en los que si falla alguna de ellas, el teorema no se cumple. 


\subsection{Aplicación de la categoría de expresiones que utiliza}

En general, en todos los LT se combinan los sistemas de representación verbal, simbólico y gráfico, aunque, dependiendo del texto, unos son más frecuentes que otros. Por ejemplo, en ambos libros de la LGE el sistema predominante es el lenguaje simbólico y, dentro del lenguaje verbal, el lenguaje específico matemático. Por el contrario, en los LT de la LOGSE y de la LOE se realizan las discusiones con mayor abundancia de lenguaje verbal, y con un estilo más coloquial que en sus predecesores.

En cuanto al sistema de representación gráfico, todos los LT presentan gráficas para ilustrar ambos teoremas (como los presentados en las Figuras 3 y 4), pero también se observan diferencias entre legislaciones: los LT de la LGE se limitan a una gráfica por teorema, mientras que en los LT de las otras legislaciones, LOGSE y LOE, se presentan varias gráficas: para ilustrar los distintos casos de la demostración del TR, para exponer varias situaciones válidas del TVM, para mostrar la necesidad de las hipótesis de los teoremas y en los ejercicios de aplicación. Esta mayor utilización de las gráficas, que además ilustran ideas de la demostración, unido al mayor uso del lenguaje verbal, aleja las justificaciones del formalismo matemático de los textos de la LGE, que tendían a una estructuración y presentación de los contenidos más propios de textos puramente matemáticos que de textos para alumnos de Educación Secundaria. Además, esta tendencia de la LOGSE y de la LOE favorece la comprensión de estos teoremas y de su justificación por parte de los alumnos.

En cuanto al análisis semántico, ya hemos mencionado algunas ideas en el análisis de los tipos de enunciado y, a excepción de los enunciados expuestos en Vicens-Vives (1979), que son de carácter geométrico, todos tienen una estructura similar, y utilizan los mismos verbos y expresiones parecidas. En ninguno de los textos se ofrecen explicaciones de las expresiones utilizadas o la simbología matemática en las pruebas de estos teoremas.

\subsection{Aplicación de la categoría de otras consideraciones generales}

En primer lugar, hemos observado si se explica el proceso de forma global antes de realizar la demostración. En ninguno de los LT analizados, ni de los dos teoremas, hacen explicación alguna. Pasan directamente del enunciado a la prueba. En dos casos (SM, 2001, 2010) se apunta que se aplica el TR al inicio de la justificación del TVM, pero no se dan más indicaciones sobre el desarrollo del proceso.

En segundo lugar, hemos observado si en el LT se establecen las consecuencias del teorema (no del hecho de haberlo justificado), como son el significado, las relaciones que se establecen con otros resultados o la utilidad. Si bien todos los LT presentan gráficas que ilustran ambos teoremas, no todos verbalizan el significado geométrico de los teoremas (los LT de LOGSE de ambas editoriales no lo hacen) y algunos (SM, 2001, 2010) acompañan los teoremas de interpretaciones físicas o situaciones reales. No obstante, en SM (2001) conviene hacer dos puntualizaciones: en el ejemplo presentado para ilustrar el TR sería interesante especificar el papel que tiene la derivada en dicho ejemplo (Figura 6), pues lo contextualizaría dentro de la situación real; por otro lado, se observa una errata en la interpretación física del TVM, ya que en la notación simbólica identifica la velocidad media en un intervalo con la velocidad instantánea. 
Para desplazarse entre dos ciudades A y B situadas a la misma altura sobre el nivel del mar, la trayectoria seguida puede ser recta y de altura constante. Pero lo normal es que la carretera que las una tenga subidas y bajadas. En los puntos donde se cambia de subir a bajar o de bajar a subir la trayectoria tiene extremos relativos. Si la trayeztoria está expresada por una función derivable, del epigrafe anterior se deduce que ia derivada en estos extremos relativos es nula. Este es el contenido del teorema de Rolle.

Figura 6. Ejemplo para el TR (SM, 2001, p. 296).

En todos los LT se presentan ejemplos de aplicación, ya sea en forma concreta y directa (todos excepto Vicens-Vives, 1979), como aplicaciones para la acotación del número de raíces en un intervalo (SM, 2010; Vicens-Vives, 1979, 1999) o para caracterizar la monotonía de funciones en un intervalo (SM, 2010; Vicens-Vives, 1999, 2009). En Vicens-Vives (1999), cuando con un ejemplo muestra cómo aplicar el TR para acotar el número de raíces, lo hace razonando por reducción al absurdo, y explicando dicho procedimiento lógico. Si bien creemos que no es el ejemplo más pertinente de aplicación de dicho procedimiento, ilustra la utilización de una técnica de demostración, algo que no suele hacerse en estos LT. En todos los LT de LOGSE y LOE que utilizan EP axiomáticos o axiomáticos y transformacionales se indica que se aplica el teorema de Weierstrass para garantizar la existencia de máximos y mínimos absolutos en el intervalo.

Atendiendo a la distinción entre enunciado y justificación, sólo dos LT etiquetan sus justificaciones con las palabras "demostración" (Vicens-Vives, 2009) y "justificación” (Vicens-Vives, 1999), lo que permite a los alumnos distinguir claramente entre su enunciado y justificación (hecho que según Ibañes y Ortega (2003), no es tan evidente en los alumnos). No así el resto de LT, en los que su distinción se reduce a un cambio tipográfico: enunciado del teorema en un recuadro, o con letra en otro formato distinto al de la demostración. Además, en Vicens-Vives (1979) no se pueden identificar las justificaciones como tal por el texto dado que están antes que los enunciados, lo que puede dificultar su reconocimiento por parte de los alumnos.

Por último, no se indican otras vías de justificación en ningún LT. Aunque en SM (2001) se identifican dos tipos de EP diferentes para el TVM (uno inductivo de varios casos y otro axiomático y transformacional), el LT no indica que se trate de dos justificaciones diferentes.

\subsection{Aplicación de las funciones de la demostración}

De las cinco funciones del modelo de de Villiers (1993), la función de comunicación está presente en todas las justificaciones, ya que el fin de los LT es comunicar el saber matemático a los estudiantes. La función de verificación aparece en todos los LT que presentan EP axiomáticos o axiomático-transformacionales, excepto en Vicens-Vives (1979), cuya forma de presentarlo no refleja una intención de verificar, sino que presenta los teoremas como conclusiones de un razonamiento. Por su parte, en Vicens-Vives (1999), que utiliza EP inductivos de 1 caso, no se aprecia una intención de verificar, sino de convencer al lector, lo que está más ligado con la función de explicación. Este tipo de EP utilizado no es aceptado por la comunidad matemática como forma de verificación. 
No hemos apreciado la función de descubrimiento en prácticamente ninguna de las justificaciones analizadas, salvo, quizá, SM (1980). Para el TR, todos los LT han establecido previamente que la derivada de una función en los extremos relativos de dicha función se anula. Sin embargo, en este LT dicho resultado es establecido en la propia demostración del TR, lo que podría considerarse un descubrimiento, aunque en el LT no se subraya como tal, y debería enunciarse a continuación como un teorema con entidad propia.

En cuanto a la función de explicación, una de las más importantes desde el punto de vista didáctico, se observan diferentes niveles en las distintas justificaciones. Por ejemplo, en Vicens-Vives (1979) hay una absoluta ausencia de función de explicación, ya que ni siquiera se indica que el razonamiento dado es una justificación del teorema enunciado posteriormente. En el resto de LT se observan indicios de explicación en las justificaciones de estos teoremas, pero son poco explícitos. Las situaciones descritas por ambos teoremas son de fácil representación gráfica, y estas representaciones se pueden utilizar para convencer de la veracidad de los teoremas desde la intuición (hecho que utiliza Vicens-Vives, 1999, que los justifica mediante representaciones gráficas). Sin embargo, el razonamiento verbal no se presenta de forma tan explicativa. Para ello sería necesario presentar las líneas generales del proceso antes de llevarlo a cabo, y justificar las elecciones de las funciones auxiliares que se utilizan para probar el TVM. En nuestro marco, otros elementos que contribuyen a la explicación son los ejemplos en contextos reales o físicos presentados por los LT de SM $(2001,2010)$. Además, SM (2001) presenta un EP inductivo de varios casos explicando el significado del teorema antes del EP axiomático-transformacional, lo que también contribuye a dicha función de explicación.

Sobre la función de sistematización, ya se ha indicado (Conejo \& Ortega, 2014) que sistematizaciones diferentes pueden confundir a los alumnos en la fundamentación de los teoremas en estudios posteriores. En este caso, no hemos encontrado errores, pero se observa una gran diversidad en cuanto a los nombres asignados a los teoremas, los enunciados, el orden considerado, las demostraciones... Esta diversidad, aunque se presenta de forma coherente en cada LT, debería evitarse.

\section{Discusión de resultados y conclusiones}

Las dos editoriales analizadas siguen directrices diferentes: SM es más constante y presenta pruebas de características similares para ambos teoremas en los tres periodos legislativos, Vicens-Vives realiza planteamientos diferentes tanto en teoría (el ejemplar de la LGE presenta estos teoremas en el capítulo dedicado al de Taylor) como en las justificaciones en cada periodo para el TVM.

Además, aunque las justificaciones utilizadas se clasifiquen dentro de una misma categoría, suelen presentar algunos matices que las hacen diferentes unas de otras. Así, se observa que los EP utilizados son similares a lo largo del tiempo, pero se aprecian una disminución en la explicitación de los pasos de las pruebas y una pérdida de formalismo en el lenguaje utilizado en las legislaciones más actuales (LOGSE y LOE). En éstas, las justificaciones son más explicativas y se realizan más observaciones orientadas hacia la comprensión de la situación y de cómo ésta no puede ser de otra forma (como forma de convencer al lector).

Se mantienen los EP axiomáticos hasta en la última legislación, hecho que contradice nuestra hipótesis de que la presencia de la demostración disminuía a lo 
largo del tiempo, al menos para estos teoremas y editoriales. Es posible que esto se deba a que estos teoremas son de fácil representación gráfica, y estas representaciones constituyan un punto de apoyo para entender los razonamientos implícitos en las demostraciones y, con ello, disminuye su complejidad. Por estas razones, se trata de buenos candidatos a ser justificados, lo que tiene una doble ventaja: por un lado, en la misma línea que apunta Hanna (1995), se provee a los alumnos de la justificación de estos enunciados, lo que facilita la comprensión. Por otro lado, son buenos ejemplos en los que formar a los alumnos en técnicas de demostración matemática, técnicas que pueden ser útiles en estudios posteriores (por ejemplo, en carreras universitarias científicas o con contenidos matemáticos de alto nivel).

Las técnicas utilizadas varían en función del tipo de EP utilizado, del grado de desarrollo de la prueba y de la sistematización utilizada en el LT. Los enunciados son similares en todos los LT excepto en Vicens-Vives (1979), aunque pensamos que debería existir mayor unificación o, al menos, cumplir ciertos requisitos: la fórmula "si...entonces" debería estar clara en el enunciado, pues permite al alumno diferenciar entre hipótesis y tesis, y se debe utilizar la expresión "al menos" u otra similar que permita al alumno deducir que existe la posibilidad de que exista alguno más. El método es un elemento que depende en gran medida de la justificación y no se puede indicar que uno sea más adecuado que otro, depende del teorema a demostrar, al igual que el estilo o el modo.

Desde el punto de vista de la teoría matemática, etiquetar teoremas y justificaciones facilita al alumno su distinción, hecho que no es tan evidente para los estudiantes, tal como descubrieron Ibañes y Ortega (2003). Además, no se suele señalar dónde terminan las demostraciones o justificaciones, lo que también puede ser motivo de confusión. Por otro lado, desde el punto de vista de la demostración, se resaltaría su interés si los propios LT mostraran indicaciones de su necesidad o señalaran su importancia. También convendría señalar las consecuencias de haber demostrado un teorema, de forma que los estudiantes perciban que la justificación no es un mero procedimiento de la teoría matemática sin aparente utilidad práctica.

Se observa una tendencia a disminuir las expresiones simbólicas, y las verbales tienden a expresarse en un lenguaje más coloquial. En todas las etapas se utilizan gráficas, pero en la LGE, los LT utilizan una única gráfica y los LT de LOGSE y LOE presentan varias, en ellas se representan los teoremas o consecuencias de ellos. Valoramos positivamente la utilización de gráficas, por su gran función explicativa y, además, abogamos por la utilización de una amplia variedad de gráficas donde se representen diferentes tipos de funciones y casuísticas que favorezcan la comprensión de estos teoremas. Además, estas gráficas deben acompañarse de explicaciones verbales, que no suelen encontrarse en los LT.

También sería conveniente la explicación del proceso de forma global antes de llevarlo a cabo, lo que facilita el seguimiento de la demostración. Esto apenas se hace en los LT y consideramos que en próximas ediciones debería incorporase. Por otro lado, todos los LT muestran ejemplos de aplicación de los teoremas, consecuencias y relaciones con otros teoremas, acciones que son positivas de cara a sistematización de la teoría.

En relación a las funciones de la demostración, las funciones de comunicación y verificación se identifican fácilmente en las justificaciones utilizadas (Conejo \& Ortega, 2013, 2014). La función de descubrimiento depende del resultado, y de la propia justificación. Sin embargo, consideramos que la función de explicación (la más 
importante según Hanna, 1995) y la de sistematización son las tareas pendientes de los LT ya que éstos deberían mostrar justificaciones explicativas, considerando esta función por delante incluso del formalismo matemático. Este formalismo debe trabajarse poco a poco, y su desarrollo es más fácil cuando se entiende el proceso. Esto es una contribución de la función de explicación. En cuanto a la función de sistematización, consideramos que se debería seguir un esquema común en todos los LT, para dar la imagen de una teoría matemática única (nombres de teoremas, orden,...) y, por supuesto, evitar cualquier error relacionado con esta función.

Por último, la utilización de un marco teórico descrito permite sistematizar un análisis que sería complejo si se quisiera atender a todos los matices diferenciadores y que, en caso contrario, resultaría demasiado superficial. Además, un análisis de este tipo permite profundizar en la epistemología de los teoremas analizados, en las diferentes sistematizaciones y tras una reflexión así, llegar al planteamiento de propuestas didácticas orientadas hacía una mejor comprensión de dichos teoremas por parte de los alumnos. Precisamente, nuestra intención es explorar esas nuevas vías, es decir, realizar una propuesta a partir de la reflexión que origina un análisis así, tarea que no es sencilla ni inmediata, e implementarla en Educación Secundaria, para comprobar si dicha propuesta es útil desde el punto de vista didáctico.

\section{Referencias}

Conejo, L., \& Ortega, T. (2013). La demostración matemática en los libros de texto de $2^{\circ}$ de B.U.P. y $1^{\circ}$ de Bachillerato de LGE, LOGSE y LOE para el concepto de límite. Fase inicial. En A. Estepa \& N. Climent (Eds.), Investigación en Educación Matemática. Comunicaciones de los grupos de investigación. XVI Simposio de la SEIEM (pp. 121-132). Baeza, Jaén: SEIEM.

Conejo, L., \& Ortega, T. (2014). Las demostraciones de los teoremas de continuidad en los libros de texto para alumnos de 17-18 años correspondientes a las tres últimas leyes educativas españolas. NÚMEROS, Revista de didáctica de las Matemáticas, 87, 5-23. Recuperado el 18 de julio de 2015 de http://www.sinewton.org/numeros/numeros/87/Articulos_01.pdf

Conejo, L., Arce, M., \& Ortega, T. (2014). Justificación de las reglas de derivación en libros de texto de cuatro editoriales desde LGE hasta LOE. En M. T. González, M. Codes, D. Arnau \& T. Ortega (Eds.), Investigación en Educación Matemática XVIII (pp. 257-266). Salamanca: SEIEM.

Conejo, L., Arce, M., \& Ortega, T. (en prensa). A case study: how textbooks of a Spanish Publisher justify results related to limits from the 70's until today. En K. Krainer \& N. Vondrová (Eds.), Proceedings of CERME9. Praga, República Checa: ERME.

de Villiers, M. (1993). El papel y la función de la demostración en matemáticas. Épsilon, 26, 15-30. Original de 1990.

Dolev, S., \& Even, R. (2015). Justifications and explanations in Israeli 7th grade math textbooks. International Journal of Science and Mathematics Education 13, Issue 2 Supplement, 309-327.

Fox, D. J. (1981). El proceso de investigación en educación. Pamplona: EUNSA. 
González, M. T. (2002). Sistemas simbólicos de representación en la enseñanza del análisis matemático: perspectiva histórica acerca de los puntos críticos. (Tesis doctoral no publicada). Universidad de Salamanca, Salamanca, España.

Hanna, G. (1995). Challenges to the Importance of Proof. For the learning of Mathematics, 15(3), 42-49. Recuperado el 18 de Julio de 2015 de http://flmjournal.org/Articles/7679867298F4CBEABE82D0ABEB5EC.pdf

Hanna, G., \& Barbeau, E. (2010). Proofs as bearers of Mathematical Knowledge. En G. Hanna, H. N. Jahnke \& H. Pulte (Eds.), Explanation and proof in mathematics: philosophical and educational perspectives (pp. 85-100). New York: Springer.

Harel, G., \& Sowder, L. (1998). Students' Proof Schemes: Results from exploratory studies. En E. Dubinski, A. Schoenfeld \& J. Kaput (Eds.), Research on Collegiate Mathematics Education III (pp. 234-283). Providence, USA: American Mathematical Society.

Ibañes, M., \& Ortega, T. (1997). La demostración en matemáticas. Clasificación y ejemplos en el marco de la Educación Secundaria. Educación matemática, 9(2), 65-104.

Ibañes, M., \& Ortega, T. (2001). Un estudio sobre los esquemas de prueba en alumnos de primer curso de bachillerato. UNO, 28, 39-60.

Ibañes, M., \& Ortega, T. (2003). Reconocimiento de procesos matemáticos en alumnos de primer curso de bachillerato. Enseñanza de las Ciencias, 21(1), 49-63. $\begin{array}{lllllll}\text { Recuperado el } 18 \text { de julio de } & \end{array}$ http://ddd.uab.cat/pub/edlc/02124521v21n1/02124521v21n1p49.pdf

Janvier, C. (1987). Traslation Processes in Mathematics Education. En C. Janvier (edt.), Problems of Representation in the Teaching and Learning of Mathematics. Hillsdale, USA: Lawrence Erlbaum Associates.

Ruiz-Berrio, J. (1976). El método histórico en la investigación histórica de la Educación. Revista Española de Pedagogía, 134, 449-475.

Schubring, G. (1987). On the Methodology of Analysing Historical Textbooks: Lacroix as textbook author. For the Learning of Mathematics, 7(3), 41-51.

Spivak, M. (1981). Calculus. Cálculo Infinitesimal. Barcelona: Reverté.

Stacey, K., \& Vincet, J. (2009). Modes of reasoning in explanations in Australian eighth-grade mathematics textbooks. Educational Studies in Mathematics, 72, 271-288.

van Ash, A.G. (1993). To prove, why and how? International Journal Mathematics Education Science and Technology, 2, 301-313.

\section{Anexo 1: Textos utilizados en el análisis}

Hernández, E., Quirós, A. y Tarrés, J. (2001). Euler. Matemáticas 2. Madrid: SM.

Pancorbo, L. (2009). Matemáticas-2. Barcelona: Vicens-Vives.

Primo, A. (1980). Matemáticas. Curso de orientación universitaria. Madrid: SM.

Ruíz, A. y Álvarez, F. (1999). Límites 2 Matemáticas. Barcelona: Vicens-Vives. 
Trillas, E. y Vila, A. (1979). Matemáticas. Espacios. Barcelona: Vicens-Vives.

Vizmanos, J.R., Hernández, J. y Alcaide, F. (2010). Matemáticas 2. Madrid: SM.

\section{Referencias a los autores}

Laura Conejo, Universidad de Valladolid (España). lconejo@am.uva.es Matías Arce, Universidad de Valladolid (España). arcesan@am.uva.es

Tomás Ortega, Universidad de Valladolid (España). ortega@am.uva.es 


\title{
An analysis of derivability theorems' justifications in textbooks from the 70's Spanish Education Law
}

\author{
Laura Conejo, Universidad de Valladolid (España) \\ Matías Arce, Universidad de Valladolid (España) \\ Tomás Ortega, Universidad de Valladolid (España)
}

This paper presents a description of a framework developed to carry out the analysis of the justifications of theorems that appear in textbooks. Our research is focused on any kind of justification that appears in textbooks of grade 12 (17-18-yearold students) from the 70's Spanish Education Law until today. Particularly, the justifications of two derivability theorems (Rolle's Theorem and mean value theorem) are examined.

This framework brings together the concepts of proof schemes (Harel \& Sowder, 1998), preformal proofs (van Asch, 1993), functions of proofs (de Villiers, 1993) and other more general aspects of proofs (Ibañes \& Ortega, 1997). The concept of personal proof scheme is adapted into the definition of textbook proof scheme. This concept together with the concept of preformal proof provide our work with the categories needed to classify the justifications shown in textbooks (inductive proof schemes of one case, of several cases or systematic inductive proof schemes; transformational proof schemes, axiomatic proof schemes and preformal proofs). Functions of proofs exhibited in textbooks were also examined and the justifications found were classified according to the general aspects of proofs given by Ibañes and Ortega (1997), like the proof techniques.

The methodology of this paper is a combination of the method of historical research on education (Ruiz-Berrio, 1976) and the research process on education (Fox, 1981). The sample used in this paper is formed by six textbooks of the three latest Spanish Educational Laws (from the Act of 1970 until the 2006 Act), two of each period. These textbooks correspond to two different publishers, one of each period to each publisher.

Previous studies about the justifications of continuity theorems suggested that the number of justifications shown in textbooks decreased over time together with the mathematical rigorous of the justifications (Conejo \& Ortega, 2014). On the contrary, the main results of this paper show that the mathematical proof of these theorems does not decrease over time but mathematical rigor does it. More graphics and contextual examples of theorems are shown in newer textbooks, which contribute to the function of explanation, the more important function of proof according to Hanna (1995). Although axiomatic proof schemes are used in all the periods, other aspects like theorems' names, order, language or explanations have changed.

We may conclude that the analysis presented in this paper is needed to systematize an analysis which could become difficult or superficial to carry out without the framework presented. Moreover, the analysis allows us to propose improvements of the teaching of mathematical proof and advanced mathematical concepts from a didactical point of view, and those improvements are our purpose for future research. 